\title{
Motor evoked potentials in the preoperative and postoperative assessment of normal pressure hydrocephalus
}

\author{
M Zaaroor, N Bleich, A Chistyakov, H Pratt, M Feinsod
}

\begin{abstract}
Motor evoked potentials and central motor conduction time (CMCT) were examined from both upper and lower limbs in patients with normal pressure hydrocephalus to find a predictor for the success of shunting procedures. The hypotheses that walking disturbances are due to pyramidal tract compression as well as the possibility that the upper limbs are affected subclinically in these patients were also studied. The study suggests that the walking disturbances are not the result of a major pyramidal tract dysfunction but probably involve the sensorimotor integration leading to normal gait. Furthermore, CMCT measured with electromagnetic motor stimulation can help in selecting the patients that will benefit from shunting. The study does not provide electrophysiological evidence of upper limb involvement in normal pressure hydrocephalus.
\end{abstract}

$(尹$ Neurol Neurosurg Psychiatry 1997;62:517-521)

Keywords: normal pressure hydrocephalus; motor evoked potentials; magnetic stimulation

The pathophysiology of the triad of symptoms (dementia, gait disturbances, and urinary incontinence) and their causative relation with the ventricular enlargement in normal pressure hydrocephalus is still enigmatic. Walking disturbances have been attributed ${ }^{1-4}$ to pressure exerted on the pyramidal tracts in the internal capsule by the distended third ventricle. This explanation was neither reviewed nor verified. Although all agree that the treatment of choice to a patient that presents the clinical picture of normal pressure hydrocephalus and the radiological finding of ventricular enlargement is a shunting procedure, only $60 \%-80 \%$ of these patients benefit from this procedure.

In this study we challenged the hypothesis that gait disturbances in normal pressure hydrocephalus are the result of pressure on the internal capsule. We also tested whether conduction to the upper limbs is affected, even without clinical manifestation. As electromagnetic stimulation of the motor cortex ${ }^{56}$ and measurement of the central motor conduction time (CMCT) allows direct evaluation of central motor function, we applied this method to consider these questions. In addition, we examined the possible utility of motor evoked potentials in prediction of outcome in patients with the clinical and radiological picture of normal pressure hydrocephalus. We report our findings and their implications on the nature of walking difficulties as well as on the criteria for patient selection.

\section{Methuds \\ CONTROLS AND PATIENTS}

This study was approved by the hospital ethics committee. Informed consent was obtained from all the volunteers and from the patients' guardians. The study was prospective and patient selection for surgery was not influenced by the results of these electrophysiological tests.

Sixteen healthy volunteers served as controls. Their age ranged from 30 to 67 . Four were women. Their height was $157 \mathrm{~cm}$ to $180 \mathrm{~cm}$. In the absence of evidence for significant age or sex effects on CMCT, we preferred a neurologically intact control group to perfect matching of age and sex.

Twenty four consecutive unselected patients with suspected normal pressure hydrocephalus were examined preoperatively. All the patients were referred to the outpatient clinic by physicians other than the operating neurosurgeons. All patients fulfilled the clinical, radiological, and known standard laboratory criteria for the diagnosis of normal pressure hydrocephalus ${ }^{7}$ including: (a) clinical presentation: either appreciable gait difficulty or the full triad of dementia, ataxia, and incontinence; (b) CT or MRI with periventricular low density or small sulci along with expansion of the ventricular system. All patients underwent lumbar puncture and isotopic cisternography with technetium (TcDTPA) as well. Patients with negative lumbar puncture or isotope cisternography with a classic clinical presentation and positive CT or MRI findings were operated on. Although some of these criteria are controversial, they still represent the standard evaluation of patients with normal pressure hydrocephalus. None of the patients showed clinical signs of upper motor neuron dysfunction in either upper or lower limbs. All 
patients presented with gait disturbances with dementia and incontinence in varying degrees.

Patients' ages ranged from 49 to 82 (mean 72) and their heights were within the range of the normal volunteers. Thirteen were women. Twenty of the 24 patients were operated on. The indications not to operate on the other four patients were medical (two) and refusal of the patient or his family (two). The patients were admitted for surgery and operated on by the departmental senior surgeons. They were evaluated with the criteria of Black $e t a l,{ }^{7}$ preoperatively and postoperatively, by the same physicians. Clinical findings correlated well with patient and family preoperative and postoperative reports. Gait was rated as: unable to walk 3; unable to walk without assistance 2 ; unsteady but able to walk unassisted 1; and without gait difficulty 0 . Standard low pressure (opening pressure of $30 \mathrm{~mm} \mathrm{H}_{2} \mathrm{O}$ ) ventriculoperitoneal shunts were implanted in all 20 patients. The motor evoked potentials were recorded preoperatively and one week postoperatively. Patients who did not show postoper-
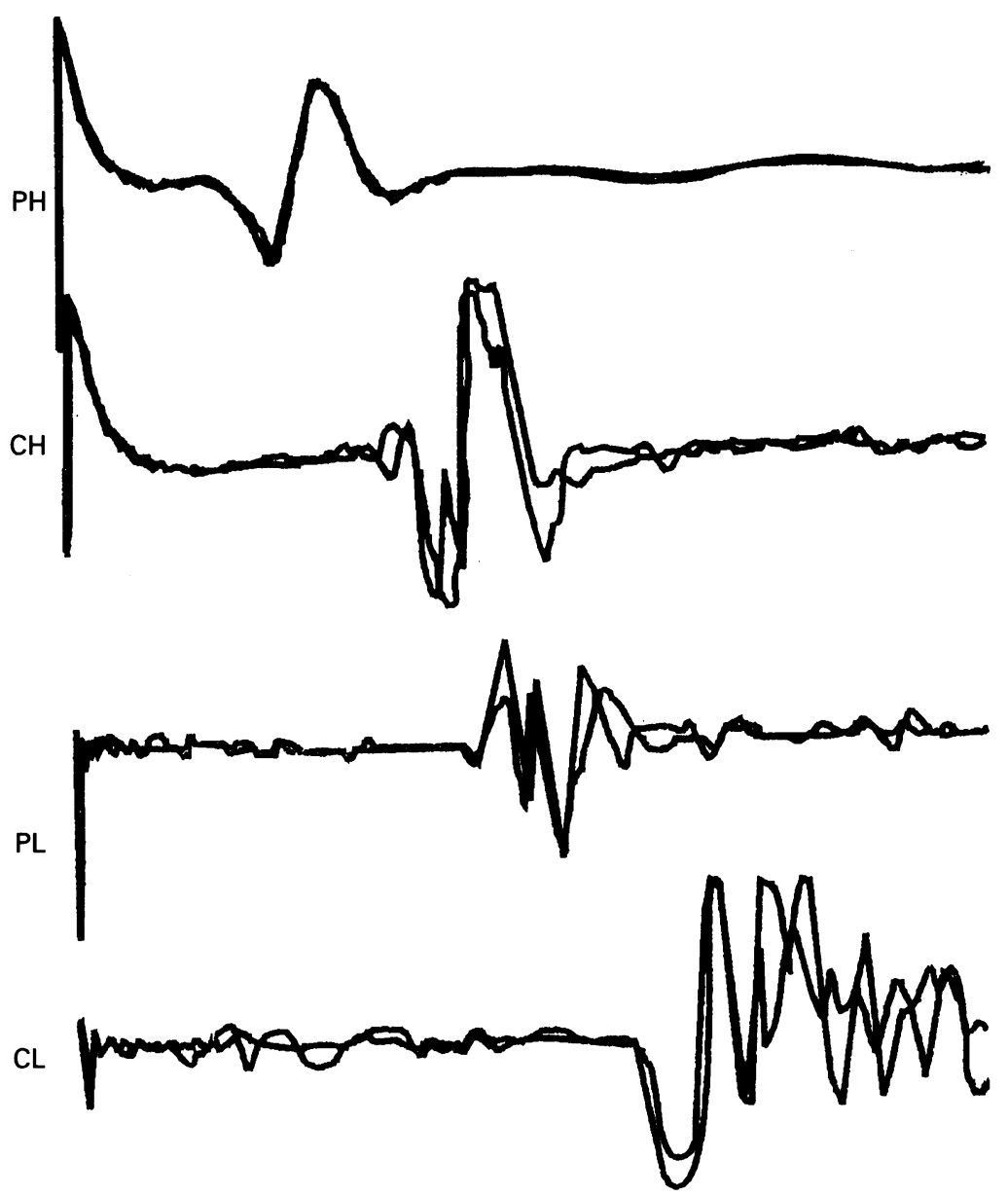

$10 \mathrm{~ms}$

Figure 1 Preoperative motor evoked potentials recorded from the thenar eminence and from the extensor digitorum brevis of a patient who subsequently improved by a shunt procedure. PH (peripheral, hand) denotes potentials recorded from the thenar eminence in response to stimulation at the level of C7. CH (central, hand) shows thenar eminence potentials after cortical stimulation. PL (peripheral, leg) stands for potentials recorded from the extensor digitorum brevis after lower lumbar stimulation. CL (central, leg) is a recording from the extensor digitorum brevis evoked by cortical stimulation. Amplitude calibration is not provided because MEP amplitudes are highly variable and were not analysed. ative gait improvement after one week were reexamined one month after operation for possible delayed recovery.

MOTOR EVOKED POTENTIAL (MEP)

Stimulation

The stimulus was a 2.5 Tesla electromagnetic field generated for about $80 \mathrm{~s}$ by a discharge of a capacitor using a $9 \mathrm{~cm}$ coil (Cadwell CEP10). The stimulus was applied over the following sites: the coronal suture to stimulate the leg area of the motor cortex and the retrocoronal paramedian to stimulate the hand area of the motor cortex; just off the midline over the level of the spinous process of $\mathrm{C} 7$ to stimulate the nerve roots to the hand as close as possible to their emergence from the spinal cord; the nerve roots to the legs were stimulated just lateral to the spinous process of L5. To standardise nerve root stimulation, we selected these anatomical markers, and verified contraction of the same muscles in all subjects, thus ensuring that error in estimating the point of stimulation along the root was uniform. The intensity of the stimulus was set to the lowest setting in which muscle potentials were recorded (between $50 \%$ to $70 \%$ ).

\section{Recording}

The MEP (the surface EMG of the movements evoked in the limbs) was recorded in the hands by surface electrodes fixed with conductive paste over the thenar eminence (active) and the base of the first digit (reference); recording from the legs was through electrodes applied over the extensor digitorum brevis muscle (active) and its insertion (reference). The MEPs were processed and displayed (negativity as a downward deflection) by an evoked potential (EP) instrument (Microshev 4000), coupled to the stimulation device. Magnetically evoked EMG from surface electrodes is highly variable in amplitude between subjects, within subjects, and between repeat recordings in the same session. The variability stems from the recording's dependence on relative positions of electrodes, muscle tissue, degree of muscle facilitation, and the variability of stimulation site with minute stimulating coil movements and threshold variability. Thus we avoided using amplitude as a dependent variable in this study. The latency between the discharge of the electromagnetic stimulus and the onset of the EMG (first deflection from baseline) was accurately determined by the cursors of the EP instrument. The time elapsed between the stimulus to the cortex and the EMG was defined as the total motor conduction time (TMCT) and the peripheral motor conduction time (PMCT) was the time between the stimulus to the nerve root and the EMG. The CMCT was the difference between TMCT and PMCT.

To ensure the same facilitation in the control study and in that of patients with normal pressure hydrocephalus, facilitation was controlled by auditory EMG feedback. Stimulus was delivered to the subject only when EMG activity was heard. The subject was instructed, 
Mean (SD) motor conduction times (ms)

\begin{tabular}{|c|c|c|c|}
\hline & $T M C T$ & $P M C T$ & $C M C T$ \\
\hline \multicolumn{4}{|l|}{ Lower limb: } \\
\hline $\begin{array}{l}\text { Normal subjects } \\
(n=16)\end{array}$ & $38.04(2.08)$ & $24 \cdot 30(1.86)$ & $13 \cdot 50(1.40)$ \\
\hline $\begin{array}{l}\text { Patients improved } \\
\text { by shunt procedure } \\
(\mathrm{n}=16)\end{array}$ & $40.02(1.90)$ & $26.40(1.50)$ & $13.50(1.70)$ \\
\hline $\begin{array}{l}\text { Patients not } \\
\text { improved by } \\
\text { shunt procedure } \\
(n=4)\end{array}$ & $46 \cdot 66(3 \cdot 24)$ & $26 \cdot 16(2 \cdot 32)$ & $19.40(0.50)$ \\
\hline $\begin{array}{l}\text { Upper limb: } \\
\text { Normal subjects } \\
(n=16)\end{array}$ & $21 \cdot 30(1 \cdot 70)$ & $13.50(1.95)$ & $7.90(1.50)$ \\
\hline $\begin{array}{l}\text { Patients improved } \\
\text { by shunt procedure } \\
(\mathrm{n}=16)\end{array}$ & $21.30(2.05)$ & $14 \cdot 20(1 \cdot 00)$ & $7 \cdot 05(2 \cdot 34)$ \\
\hline $\begin{array}{l}\text { Patients not } \\
\text { improved by } \\
\text { shunt procedure } \\
(n=4)\end{array}$ & $24.00(3.30)$ & $14.50(1.00)$ & $9 \cdot 40(4 \cdot 08)$ \\
\hline
\end{tabular}

when possible, to contract the muscle minimally so that EMG could just be heard. This assured a stable state of facilitation without interference with clear recording of the EP.

\section{STATISTICAL ANALYSIS}

The criterion for normal latency of the MEP was a value within 3 SD of the average control value.
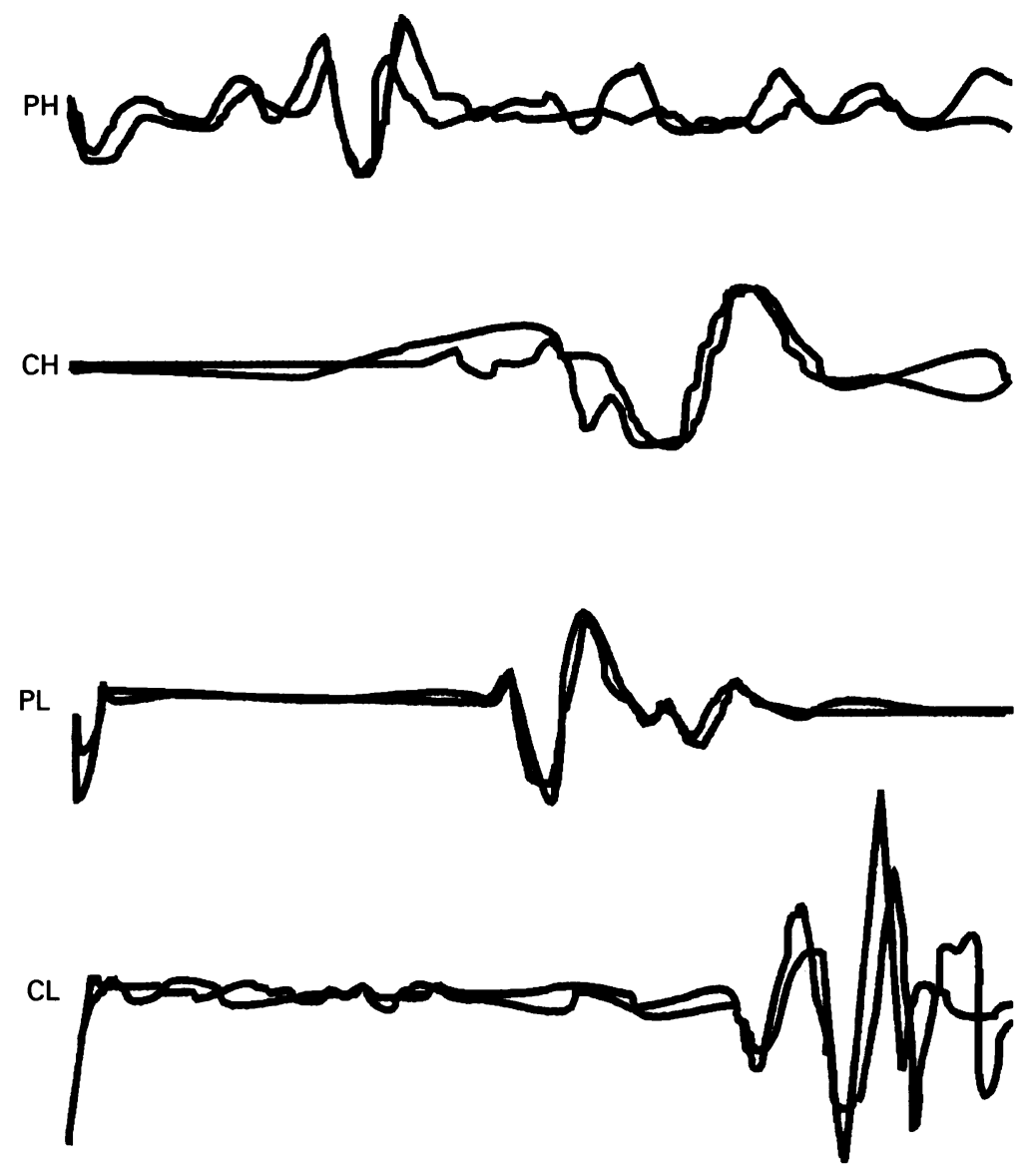

$10 \mathrm{~ms}$

Figure 2 Preoperative motor evoked potentials recorded from the thenar eminence and from the extensor digitorum brevis of a patient who subsequently did not improve by a shunt procedure. Note the increased central motor conduction time to lower limb activation compared with the patient who improved by the shunt procedure. For explanation of abbreviations see fig 1.
In correlating clinical findings with MEP latencies and CMCT, Pearson multiple regression correlations $>0.6$ were considered significant. In comparing MEP findings between subject groups, $\mathrm{P}$ values $<0.05$ by Student's $t$ test were considered significant.

Results

Motor evoked potentials from normal subjects and from patients with normal pressure hydrocephalus that improved immediately after surgery were indistinguishable. Figure 1 shows such normal motor evoked potentials.

\section{NORMAL SUBJECTS}

The table gives the means (SD) of the TMCTs, PMCTs, and CMCTs which were obtained from the normal subjects.

\section{PATIENTS WITH NORMAL PRESSURE}

HYDROCEPHALUS

Gait in 16 of the 20 patients improved immediately after surgery, whereas four remained unchanged despite a patent shunt. Duration of symptoms in patients of either group was not different, and patients from all groups showed the same variations in severity of dementia, incontinence, and ataxia. The two major groups were clinically indistinguishable preoperatively.

In the group of patients with normal pressure hydrocephalus that improved after the shunt procedure, preoperative and postoperative central conduction times were within the norm and did not show any significant difference $(P>0.05)$ as a result of the shunting procedure (table, fig 2 ). There were no significant changes $(P>0.05)$ between preoperative and postoperative values in CMCT to either hands or legs (table). In most patients peripheral conduction time was within normal values. In three patients the peripheral conduction times to the leg or the hand were abnormally long and the TMCTs were also long. The associated CMCTs were within normal limits. The gait disturbance in these three patients was most probably not secondary to peripheral neuropathy. After the shunting procedure these patients showed improvement in their gait and other normal pressure hydrocephalus symptoms, even though their prolonged peripheral conduction time persisted.

All four patients that showed no improvement after the shunting procedure had abnormal preoperative CMCTs to the legs whereas CMCTs to the hand and the peripheral conduction to hands and legs were normal (table, fig 3). There were no significant differences in latencies between preoperative and postoperative measures up to one month of follow up.

The number of patients that showed no improvement was small, and as a result statistical tests were confined to comparison with normal limits. There was a significant difference in the MEP results preoperatively between patients with successful shunting and those with no improvements after shunting (fig 3 ). There was a significant difference between 


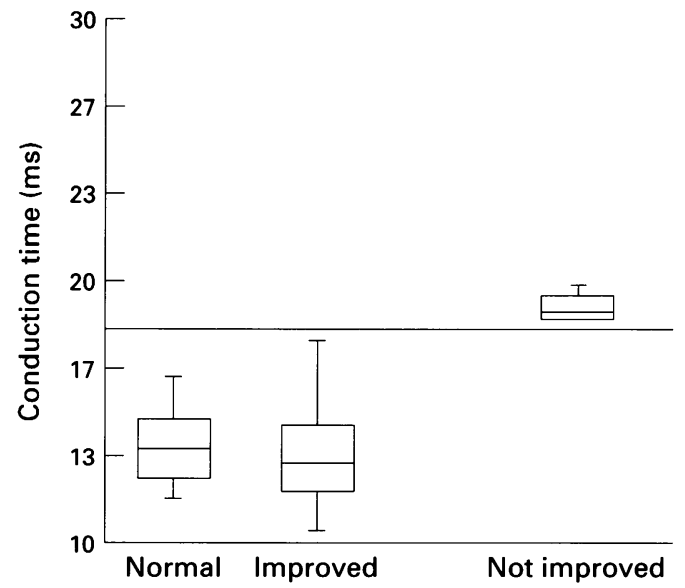

Figure 3 Central lower limb conduction times in normal subjects, in patients who improved by the shunt procedure, and in those not improved. The thick horizontal line is the upper limit of the norm. The boxes represent the range within which $50 \%$ of the values were found ( 25 percentiles from the median marked by the horizontal line through each box). Error bars denote the 99th percentile range. Note that all patients who were not improved by the shunt procedure had abnormally prolonged central lower limb conduction times preoperatively.

the normative subjects' CMCT and data from patients with unsuccessful shunting procedures.

\section{Discussion}

The prediction of outcome of shunting procedures in patients with normal pressure hydrocephalus is based either on subjective impressions of the clinical presentation of the triad of dementia, gait disturbances, and urinary incontinence, and on complimentary tests $^{812}$ such as CSF dynamics or changes in the cerebral blood flow after CSF drainage, which provide only corroborative evidence of improvement. The clinical picture may stem from various aetiologies and cannot, therefore, serve as a reliable indication for surgery, even when associated with ventricular enlargement. The ability to predict success of shunt procedures in patients with suspected normal pressure hydrocephalus ranges between $60 \%$ and $80 \%{ }^{7}$ Gait disturbance is a cardinal symptom in normal pressure hydrocephalus and its amelioration may serve as a criterion for success of the shunting procedure, especially if it dominates the clinical picture or precedes the impaired mentation. ${ }^{13}$ Although many attempts have been made to explain the cause of ventricular enlargement in normal pressure hydrocephalus the pathophysiology of the symptoms and their relation to hydrocephalus have not been reliably explained. The old theory of Yakovlev ${ }^{15}$ that gait disturbance is caused by compression of the internal capsule fibres by the distended third ventricle may still be found in basic textbooks. ${ }^{4}$ Yakovlev tried to explain paraplegia in children with enlarged ventricles but never attempted to explain gait disturbances in patients with normal pressure hydrocephalus that do not show clinical signs of upper motor neuron lesions or lower limb weakness. In addition the anatomical basis of his theory was never established.
In this study we tested Yakovlev's hypothesis physiologically. We elected to use electromagnetic stimulation of the motor cortex to assess conduction along its efferent pathways. Although the exact site and nature of the cortical activity evoked by magnetic stimulation is still unclear ${ }^{56}$ it may be stated safely that the generated impulses are conveyed along the pyramidal pathways. ${ }^{5}$

The patients that were improved by shunting all had normal preoperative CMCTs whereas those with delayed preoperative CMCTs did not benefit from surgery. Moreover, the shunting procedure had no effect on the CMCT to the leg nor to the hand, regardless of whether the patient's gait improved or not.

Our results do not support the theory of pyramidal tract involvement in the disturbed gait of patients with normal pressure hydrocephalus. Pressure on the pyramidal tracts can theoretically cause demyelination (expected to manifest in latency changes), axonal loss (manifesting in amplitude diminution and usually latency prolongation), or both. Thus latency measures are the single comprehensive measure to detect the effect of pressure on the pyramidal tract. The MEP is not acclaimed as the ultimate test for the integrity of the motor system, and its sensitivity in detecting lesions along the motor system is limited. However, in our study patients were very sharply divided into a group that had no MEP abnormality (all of whom improved) and a group that were uniformly abnormal in their MEP (all of whom did not improve). The uniform division to these two groups and the pronounced difference (3 SD) between groups strongly indicate that patients with documented involvement of the motor system according to MEP are a distinct group that does not benefit from shunting. Furthermore, these results strongly suggest that conduction along the central motor pathway is not affected in patients with normal pressure hydrocephalus.

It may be argued that involvement of the pyramidal tract, as evidenced by prolonged CMCT, may represent a final irreversible stage of normal pressure hydrocephalus. Alternatively, the unchanged CMCT after shunting, together with the finding that the CMCT to the hand was found not to be involved in any extent in either group, do not support such an explanation. The reversible gait disturbance may thus be either a form of gait apraxia or ataxia of non-pyramidal origin, and its cerebral mechanism and localisation remain enigmatic. Either way, a patient presenting a clinical picture of normal pressure hydrocephalus with prolonged CMCT seems to be a poor candidate to benefit from shunting.

In conclusion, our results clearly show that a normal preoperative CMCT to the legs that remains unchanged after surgery was found in all the patients with normal pressure hydrocephalus who improved by shunting. In addition, the results show that prolonged CMCT to the legs was found in all the patients who did not improve. Thus patients with normal 
pressure hydrocephalus with CMCT within the normal range have a better chance of benefiting from shunt surgery than those with delayed CMCT. Evidence for pyramidal tract involvement as seen by MEP in patients with suspected normal pressure hydrocephalus should sober the expectations from shunting. In our series, the MEP, and particularly CMCT, proved to be a good predictor of outcome and can thus be added to the armamentarium of preoperative evaluation. This procedure proved to be an objective, simple, non-invasive, and reproducible test.

1 Adams RD, Fisher CM, Hakim S, Ojemann RD, Swee WH. Symptomatic occult hydrocephalus with "normal" cerebrospinal fluid pressure. $N$ Engl $f$ Med 1965;273 $117-26$.

2 Hakim S. Some observation on CSF pressure hydrocephalic syndrome in adults with "normal" CSF pressure (recognition of a new syndrome). Bogota, Colombia: Javeriana University School of Medicine, 1964. (Thesis No 957, English translation.)

3 Fisher CM. Hydrocephalus as a cause of disturbances of gait in elderly. Neurology 1982;32:1358-63.

4 Adams RD and Victor M. Disturbances of cerebrospinal fluid circulation including hydrocephalus and meningial fluid circulation including hydrocephalus and meningial
reaction. In: Adams RD, Victor M, eds. Principles of neu- rology. 5th ed. New York: McGraw Hill ,1993:545-6.

5 King PJL, Chiappa KH. Motor evoked potentials. In: Chiappa $\mathrm{KH}$, ed. Evoked potentials in clinical medicine. 2nd ed. New York: Raven, 1990:509-62.

6 Mills KR, Murray NM, Hess CW. Magnetic and electrical transcranial brain stimulation: physiological mechanisms and clinical applications. Neurosurgery 1987;20:164-8.

7 Black PMcL, Ojemann RG, Tzouras A. CSF shunts for dementia, incontinence and gait disturbance. Clin Neurosurg 1984;32:632-51.

8 Bannister R, Gilford E, Kocen R. Isotope encephalography in the diagnosis of dementia due to communicating hydrocephalus. Lancet 1967;ii:1014-7.

9 Hartmann A, Alberti E. Differentiation of communicating hydrocephalus and presenile dementia by continuous recording of cerebrospinal fluid pressure. $f$ Neurol Neurosurg Psychiatry 1977;40:630-40.

10 Hussey F, Schanzer B, Katzman R. A simple constant infusion manometric test for measurement of CSF absorpsion manometric test for measurement of CSF absorp-
tion II. Clinical studies. Neurology (Minneapolis) 1970;20: tion II. Clin.

11 Laws ER Jr, Mokari B. Occult hydrocephalus: results of shunting correlated with diagnostic tests. Clin Neurosurg 1977;24:316-33.

12 Brown DG, Goldensohn ES. The electroencephalogram in normal pressure hydrocephalus. Arch Neurol 1973;29: $70-1$

13 Fisher CM. The clinical picture in ocult hydrocephalus. Clin Neurosurg 1977;24:270-84.

14 Ojemann KG, Black PM. Evaluation of patient with dementia and treatment of normal pressure hydrocephalus. In: Wilkins RH, Rengachary SS, eds. Neurosurgery. Vol I. New York: McGraw-Hill, 1985:316.

15 Yakovlev PI. Paraplegias of hydrocephalics. American Fournal of Mental Deficiency 1947;LI:561-76. 